Nota de investigación

\title{
Análisis de perfil de textura de frutos partenocarpicos de mango cv. 'Ataúlfo'
}

Yanik Ixchel Maldonado-Astudillo ${ }^{1,2}$

Ángel Cantú-Guzmán ${ }^{1}$

Ricardo Salazar ${ }^{3}$

Javier Jiménez-Hernández ${ }^{1,2 \S}$

${ }^{1}$ Facultad de Ciencias Químico Biológicas-Universidad Autónoma de Guerrero-Ciudad Universitaria sur. Av. Lázaro Cárdenas s/n, Col. la Haciendita, Chilpancingo de los Bravo, Guerrero, México. CP. 39087. Tel. 747472 5503. (ángel_acg142@ @otmail.com). ${ }^{2}$ Maestría en Competitividad y Sustentabilidad-Universidad Autónoma de Guerrero. Calle Pino s/n, Col. El Roble, Acapulco, Guerrero, México. CP. 39640. Tel. 744 4877740. (yaixma@gmail.com; jjimenez@uagro.mx). ${ }^{3}$ CONACyT-Universidad Autónoma de Guerrero, Av. Javier Méndez Aponte núm. 1, Fracc. Servidor Agrario, Chilpancingo de los Bravo, Guerrero, México. CP. 39070. Tel. 7474719310.

§Autor para correspondencia: jjimenez@uagro.mx.

\section{Resumen}

Los frutos sin semilla son llamados partenocarpicos, en el mango poseen un tamaño de dos a tres veces menor que los frutos normales y un alto contenido de azúcares. Son llamados 'mango niño', tienen un bajo valor económico debido a que no reúnen los estándares del mercado nacional e internacional. El objetivo de este estudio fue caracterizar frutos partenocarpicos de mango cv. 'ataulfo'; a través, de un análisis de perfil de textura (TPA), pérdida de peso y contenido de sólidos solubles (TSS). Los frutos partenocarpicos (PF) mostraron una disminución en las características texturales hasta alcanzar su madurez. Sin embargo, en los frutos no partenocarpicos (NPF) la disminución fue menos pronunciada. Los PF mostraron una textura más suave y los parámetros más significativos fueron la dureza y la masticabilidad. La pérdida de peso y contenido de TSS fueron mayores durante el almacenamiento en PF, indicando pulpa con mayor dulzor al final del almacenamiento. Los resultados proveen información importante sobre los usos potenciales del mango niño. Se sugiere que la pulpa suave y dulce puede ser utilizada en aplicaciones tales la preparación de jarabes para preparar bebidas o concentrados de mango.

Palabras clave: análisis de perfil de textura, fruto partenocarpico, mango.

Recibido: julio de 2019

Aceptado: agosto de 2019 
La textura es definida como la manifestación sensorial de la estructura del alimento y la manera en la cual actúan las fuerzas aplicadas, representa la conjunción de atributos mecánicos, geométricos y superficiales de un producto censado a través de sensores mecánicos, de tacto, visuales y auditivos (Szczesniak, 1963; Torres-González et al., 2015). No obstante, la textura puede ser relacionada con la deformación, desintegración y el flujo de alimentos cuando se les aplica una fuerza (Bourne, 2002).

La textura es un atributo sensorial crítico que puede dominar la calidad del producto como los frutos. Desde el punto de vista del consumidor, la textura es generalmente referida como un indicador de la calidad comestible y frescura de los frutos, así como el principal factor que determina la aceptación del producto (Valente et al., 2011). Uno de los principales problemas de los frutos como el mango es la perdida de textura (Sirijariyawat et al., 2012). Actualmente la comercialización de frutos mínimamente procesados es una opción comercial atractiva; sin embargo, el ablandamiento y pérdida de textura es un problema en frutos y vegetales rebanados.

El mango (Mangifera indica L.) es uno de los frutos tropicales más populares del mundo (Mitcham, 1992). Su pulpa (mesocarpio) rápidamente pierde consistencia durante la maduración, por lo que debe consumirse antes de que alcance su madurez total y desarrolle características indeseables al consumidor (Maldonado-Astudillo, 2016).

Hasta ahora, existen pocos reportes sobre la textura de frutos partenocarpicos (Banjongsinsiri et al., 2004; Valente, 2011; Sirijariyawat et al., 2012; Ellong et al., 2015; Alvis-Bermudez et al., 2016). Estos frutos no poseen semilla y poseen un tamaño dos a tres veces menor que los frutos normales, así como un alto contenido de sólidos solubles. Estos frutos tienen un bajo precio en el mercado debido a que no reúnen los atributos de las normas nacionales e internacionales. Por lo que su comercialización está limitada al mercado nacional y su producción es aprovechada parcialmente (Pérez-Barraza, 2007).

En México, la partenocarpia ha sido reportada en las principales zonas productoras de mango de los estados de Nayarit, Guerrero y Chiapas y se ha observado un incremento de este fenómeno (Leyva-Mayo et al., 2016). La ausencia de semilla en los frutos de mango afecta considerablemente el tamaño, llegando a ser tres veces menor que los frutos con semilla. El tamaño de los frutos afecta su aceptación y comercialización, lo cual genera pérdidas económicas en la producción de mango cv. 'Ataulfo'. El objetivo de este estudio fue caracterizar frutos partenocarpicos de mango cv. 'Ataulfo'; a través, de análisis de perfil de textura, pérdida de peso y contenido de sólidos solubles y generar información que permita proponer aplicaciones potenciales para estos frutos.

\section{Material biológico}

Frutos de mango cv. 'Ataulfo' en el mismo estado de madurez (madurez fisiológica) fueron cosechados de un huerto de Atoyac de Álvarez (Costa Grande, Guerrero) y llevados al laboratorio. Posteriormente fueron seleccionados, lavados y secados para ser usados en el estudio. 100 unidades experimentales (50 frutos partenocárpicos (PF) y 50 frutos no partenocarpicos (NPF) fueron almacenados a $24 \pm 1{ }^{\circ} \mathrm{C}$ en una incubadora de baja temperatura durante cinco días. Las pruebas fueron realizadas cada $24 \mathrm{~h}$ durante el almacenamiento. 


\section{Análisis físicos}

Se utilizó un muestreo no destructivo con 10 frutos como unidad experimental, para evaluar el peso con una balanza digital (Ohaus ${ }^{\circledR}$, USA) con una sensibilidad de $0.1 \mathrm{~g}$. El estudio de perfil de textura (TPA) fue realizado con un analizador de textura (TA.XT plus Texture Technologies Corp, Scarsdale, NY). Los frutos fueron comprimidos dos veces con una sonda de compresión de $75 \mathrm{~mm}$ de diámetro con una celda de carga de $25 \mathrm{~kg}$ a una velocidad de $2 \mathrm{~mm} \mathrm{~s}^{-1}$, hasta lograr $20 \%$ de compresión del diámetro transversal del fruto, con un intervalo entre compresiones de $10 \mathrm{~s}$.

A partir de los resultados de las dos compresiones, fueron calculados los siguientes parámetros: dureza $(\mathrm{N})$, fuerza necesaria para generar una deformación de la muestra; fracturabilidad $(\mathrm{N})$, fuerza necesaria para fracturar la muestra, adhesividad $\left(\mathrm{N} \mathrm{s}^{-1}\right)$, trabajo necesario para superar las fuerzas de atracción entre la superficie del alimento y la superficie de la sonda, elasticidad $\left(\mathrm{N} \mathrm{s}^{-1}\right)$, tasa a la que un material deformado vuelve a su condición inicial después de que se retira la fuerza de deformación, cohesividad $(\mathrm{N})$, es el grado en que un material puede deformarse antes de romperse, masticabilidad $(\mathrm{N})$, energía requerida para desintegrar un alimento sólido listo para masticar; y resiliencia $(\mathrm{N})$, capacidad de recuperación de un material para regresar a su estado original después de la primera compresión (Szczesniak, 2002).

\section{Análisis químicos}

Se aplicó un muestreo destructivo con 10 frutos como unidad experimental para Sólidos Solubles Totales (TSS, ${ }^{\circ}$ Brix). Los TSS se midieron en apego a la NMX-F-103-1982 utilizando un refractómetro digital $\mathrm{ATAGO}^{\circledR}$ previamente calibrado con agua destilada, en el cual se colocó una gota de jugo de cada muestra. Las mediciones se realizaron por triplicado y los resultados se expresaron en ${ }^{\circ}$ Brix.

\section{Análisis estadístico}

los resultados son reportados como el promedio \pm desviación estándar $(n=10)$. Se realizó una prueba de Anova y una prueba posterior de Tukey HSD para determinar diferencias estadísticamente significativas $(p<0.05)$ de las características de los frutos usando el paquete estadístico SPSS ver. 22 (IBM Inc., USA).

\section{Discusión}

\section{Cambios de textura}

La Figura 1a, 1b muestra el análisis de perfil de textura de frutos partenocarpicos (PF) y no partenocarpicos (NPF) de mango cv. 'Ataulfo' durante 5 días de almacenamiento a $24{ }^{\circ} \mathrm{C}$. Se observaron diferencias significativas $(p \leq 0.05)$ en dureza y masticabilidad, siendo los PF los presentaron menor dureza y masticabilidad. La elasticidad, cohesividad y resilencia no mostraron diferencias significativas $(p \leq 0.05)$. Sin embargo, ambos tipos de frutos mostraron la misma tendencia de disminución de sus parámetros texturales durante el almacenamiento. Los cambios texturales son debidos principalmente al proceso de maduración, durante el cual se realiza la hidrólisis de almidón y otros componentes estructurales como las pectinas, lo cual impacta de manera importante en la textura de los frutos (Huber, 1983). 

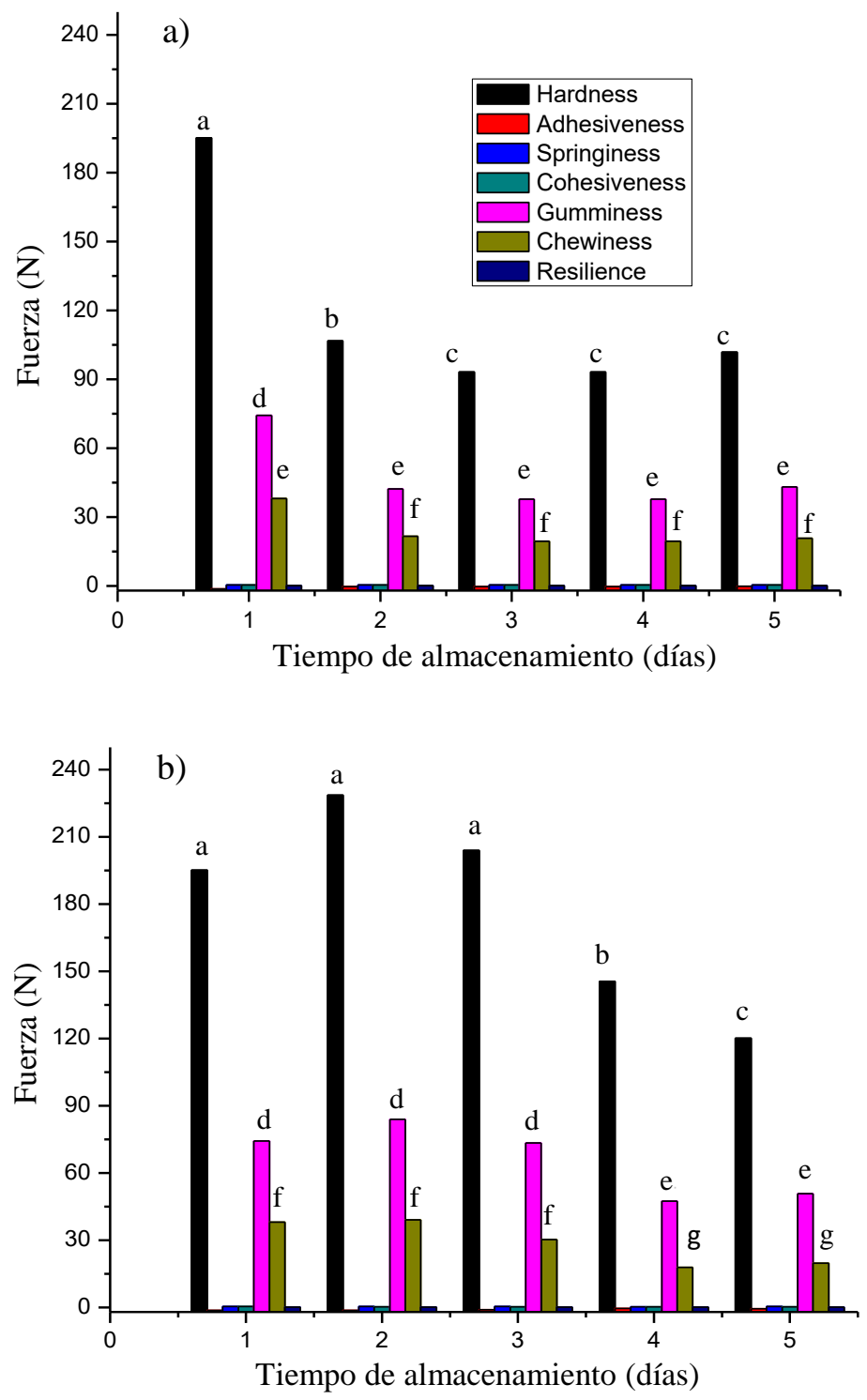

Figura 1. Análisis de perfil de textura en frutos partenocarpicos (a) y no partenocarpicos (b) de mango cv. 'Ataulfo' durante 5 días de almacenamiento a $24{ }^{\circ} \mathrm{C}$.

De acuerdo con Szczesniak (1995), se recomienda que se haga referencia a la masticabilidad para los productos sólidos. En este estudio, los parámetros texturales de los frutos de mango más significativos fueron la dureza y masticabilidad.

La mayor dureza observada en NPF es parcialmente debido a que los frutos tienen tres fracciones (epicarpio, mesocarpio y endocarpio), mientras que PF solo tienen dos (epicarpio y mesocarpio) (Figura 2). El endocarpio está vacío. Esta condición afecta negativamente la dureza de los frutos durante la compresión.

De acuerdo con estudios previos, los frutos partenocarpicos de mango tienen un alto contenido de azucares solubles y menor contenido de almidón que los frutos no partenocarpicos. El incremento de TSS está relacionado con el ablandamiento de la pulpa (Leyva-Mayo, 2016). 


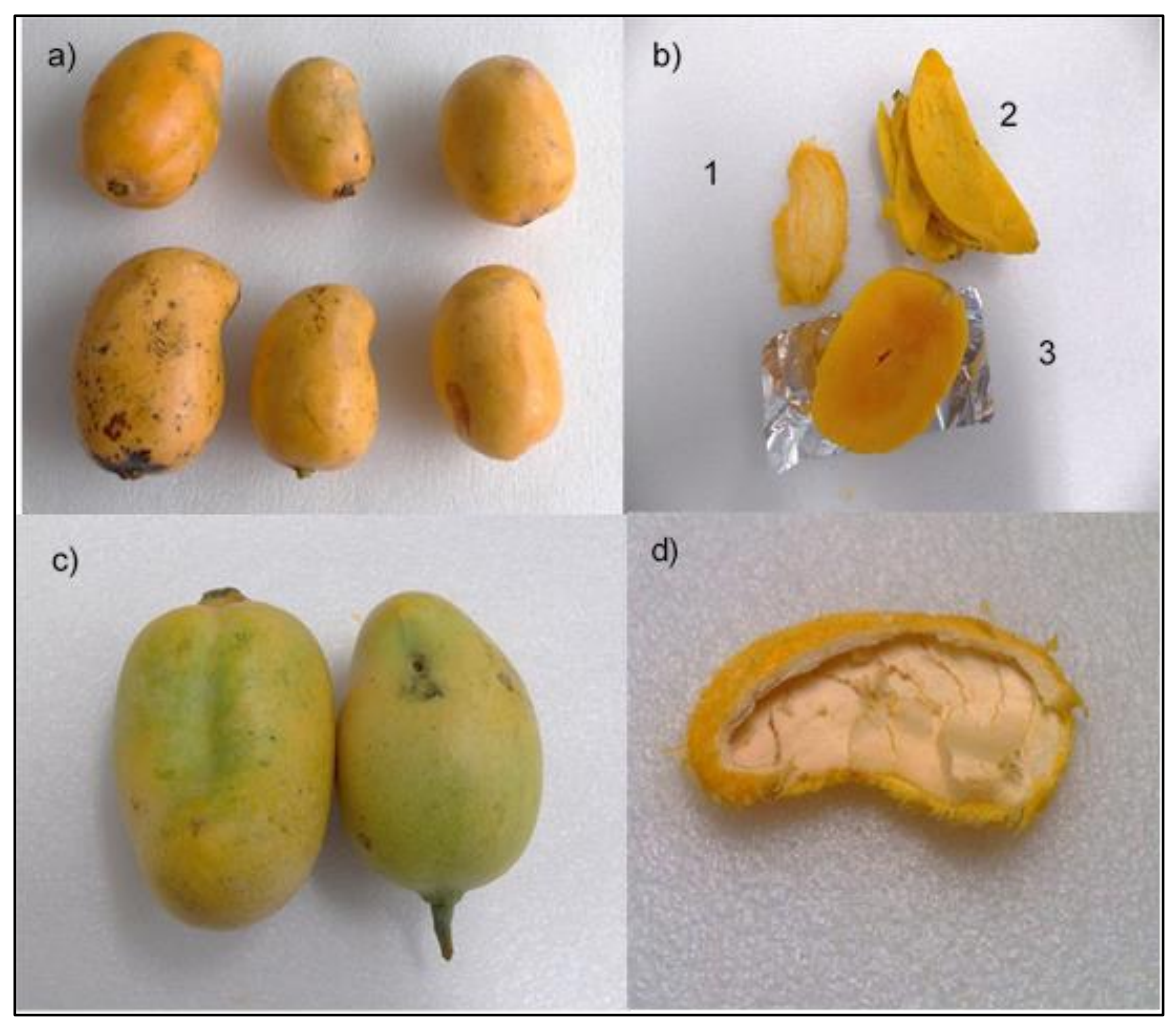

Figura 2. a) frutos partenocarpicos de mango cv. 'Ataulfo'; b) fracciones de los frutos: 1 endocarpio (hueso); 2 exocarpio (piel); 3 mesocarpio (pulpa); c) frutos partenocarpicos de mango en madurez fisiológica; d) endocarpio sin semillas.

\section{Cambios físicos y químicos}

Se observó un incremento en la pérdida de peso y en el contenido de TSS durante el almacenamiento en ambos frutos (Cuadro 1), siendo los PF los que observaron mayores valores de TSS y NPF los que presentaron la mayor pérdida de peso.

Cuadro 1. Pérdida de peso y contenido de solidos solubles en frutos de mango cv. 'Ataulfo' almacenados a $24{ }^{\circ} \mathrm{C}$ durante 5 días.

\begin{tabular}{cccccc}
\hline \multirow{2}{*}{$\begin{array}{c}\text { Tiempo de } \\
\text { almacenamiento } \\
\text { (días) }\end{array}$} & \multicolumn{2}{c}{ Frutos partenocarpicos } & & \multicolumn{2}{c}{ Frutos no partenocarpicos } \\
\cline { 6 - 7 } \cline { 5 - 6 } & TSS $\left({ }^{\circ}\right.$ Brix $)$ & $\begin{array}{c}\text { Pérdida de peso } \\
(\%)\end{array}$ & & TSS $\left({ }^{\circ}\right.$ Brix $)$ & $\begin{array}{c}\text { Pérdida de peso } \\
(\%)\end{array}$ \\
\hline 1 & $25.6 \pm 0.5 \mathrm{~d}$ & $0 \pm 0.8 \mathrm{e}$ & & $17.86 \pm 0.6 \mathrm{f}$ & $0 \pm 0.5 \mathrm{e}$ \\
2 & $26.2 \pm 0.5 \mathrm{~d}$ & $4.5 \pm 0.2 \mathrm{~d}$ & & $19.3 \pm 0.4 \mathrm{f}$ & $5 \pm 0.2 \mathrm{c}$ \\
3 & $28.8 \pm 0.5 \mathrm{c}$ & $6.7 \pm 0.5 \mathrm{c}$ & & $20.8 \pm 0.3 \mathrm{f}$ & $7.2 \pm 0.7 \mathrm{~b}$ \\
4 & $31 \pm 0.5 \mathrm{~b}$ & $8.3 \pm 0.7 \mathrm{~b}$ & & $22.3 \pm 0.7 \mathrm{e}$ & $9.4 \pm 0.4 \mathrm{~b}$ \\
5 & $33.5 \pm 0.5 \mathrm{a}$ & $9.1 \pm 0.2 \mathrm{~b}$ & & $25.3 \pm 0.2 \mathrm{~d}$ & $10.6 \pm 0.3 \mathrm{a}$ \\
\hline
\end{tabular}

Promedio de 10 repeticiones \pm desviación estándar. Las letras indican diferencias significativas entre los frutos partenocarpicos y no partenocarpicos. 


\section{Conclusiones}

Los resultados obtenidos en este estudio muestran que los frutos partenocarpicos presentaron menor dureza y masticabilidad y mayor contenido de azúcares que los frutos no partenocarpicos durante las condiciones de almacenamiento, lo cual muestra que el mango niño posee pulpa más suave y dulce que los frutos normales. Estos resultados proveen información importante para proponer usos potenciales como ingrediente para elaborar bebidas o jarabes.

\section{Agradecimientos}

Los autores(as) agradecen el financiamiento de la Universidad Autónoma de Guerrero: proyecto de investigación semilla 2014.

\section{Literatura citada}

Alvis-Bermúdez, A.; García-Mogollon, C. y Dussán-Sarria, S. 2016. Cambios en la textura y color en mango (Tommy Atkins) presecado por deshidratación osmótica y microondas. Información Tecnológica. 27(2):31-38.

Banjongsinsiri, P.; Kenney, S. and Wicker, L. 2004. Texture and distribution of pectic substances of mango as affected by infusion of pectinmethylesterase and calcium. J. Sci. Food Agric. 84(12):1493-1499.

Bourne, M. C. 2002. Food texture and viscosity: concept and measurement. Second (Ed.). Academic Press. San Diego. 15 p.

Ellong, E. N.; Adenet, S. and Rochefort, K. 2015. Physicochemical, nutritional, organoleptic characteristics and food applications of four mango (Mangifera Indica) varieties. Food Nutr. Sci. 6(2):242-253.

Huber, D. J. 1983. The role of cell wall hydrolases in fruit softening (texture changes, food quality). Hort. Rev. 5:169-219.

Leyva-Mayo, A.; Noriega-Cantú, D. H.; Pérez-Barraza, M. H.; González-Mateos, R. y HernándezPereyda, J. 2016. Distribución geográfica de frutos partenocárpicos en mango cv. 'Ataúlfo' en la costa de Guerrero. Rev. Foro estudio sobre Guerrero. 2:1-5.

Maldonado-Astudillo, Y. I. Navarrete-García, H. I., Ortiz-Morales, O. D., Jiménez-Hernández, J., Salazar-López, R., Alia-Tejacal, I., Álvarez-Fitz, P. 2016. Propiedades físicas, químicas y antioxidantes de variedades de mango crecidas en la costa de Guerrero. Rev. Fitotec. Mex. 39(3):207-214.

Mitcham, E. J. and Mcdonald, R. E. 1992. Cell wall modification during ripening of 'Keitt' and 'Tommy Atkins' mango fruit. J. Am. Soc. Hortic. Sci. 117(6):919-924.

Pérez-Barraza, M. H.; Vázquez-Valdivia, V. y Osuna-García, J. A. 2007. Incidencia de frutos partenocárpicos en mango 'Ataulfo' en huertos comerciales de Nayarit. Rev. Chapingo Ser. Hortic. 13(2):149-156.

Sirijariyawat, A.; Charoenrein, S. and Barrett, D. M. 2012. Texture improvement of fresh and frozen mangoes with pectin methylesterase and calcium infusion. J. Sci. Food Agric. 92(13):2581-2586.

Szczesniak, A. S. 1963. Classification of textural characteristics. J. Food Sci. 28(4):385-389.

Szczesniak, A. S. 1995. Profile analysis methodology interpretation clarified. J. Food Sci. 60(7):1-7. 
Szczesniak, A. S. 2002. Texture is a sensory property. J. Food Sci. Food Quality Preference. 13(4):215-225.

Torres-González, D.; González-Morelos, K. J. y Acevedo-Correa, D. 2015. Análisis de perfil de textura en frutas, productos cárnicos y queso. Recitela. 12(2):64-75.

Valente, M.; Ribeyre, F.; Self, G.; Berthiot, L. and Assemat, S. 2011. Instrumental and sensory characterization of mango fruit texture. J. Food Quality. 34(6):413-424. 